\title{
RFID based Audio Tour Guide with Monitoring Functions
}

\author{
Karishma Vora ${ }^{1}$, Meghana Vishwanath ${ }^{2}$, \\ Naina Mehta ${ }^{3}$, Soumya Belur ${ }^{4}$ \\ UG Student , \\ Dept. of Electronics and Instrumentation, \\ JSS Academy of Technical Education, Bengaluru, \\ Karnataka, India 1,2,3,4
}

\author{
Dr. Jayadevappa ${ }^{5}$ \\ Assoc. Professor, \\ Dept. of Electronics and Instrumentation , \\ JSS Academy of Technical Education, \\ Bengaluru, Karnataka, India ${ }^{5}$
}

\begin{abstract}
Tourism plays a critical role in the economy of a country hence it becomes extremely important that the correct information is delivered to the tourists. There is an existing location aware audio travel system which uses GPS to tell the location of the listener. But GPS is proved to be extremely inaccurate which can cause trouble to the tourist. The proposed system is divided into two parts: The moving robot and a RFID communication. The moving robot scans the $Q R$ code through the RaspberryPi Picamera and follows it. If a human carries the QR Code, the robot will follow the human to any location.The second part uses Radio Frequency Identification (RFID) integrated which relies on Radio Waves. Each location or artifact is embedded with a NRF24L01 module, which is a radio frequency transceiver, that acts as a transmitter, and the robot acts as a receiver. Each module sends a unique radio signal. Based on the signals received by the receiver unit relevant information such a text, image, audio can be delivered to the user. In addition to an audio tour guide we also believe it is necessary to maintain a log of every individual who enters a designated area that is part of the tour. To maintain these logs, we propose using NFC sensors linked to a specified server that can be accessed whenever required.
\end{abstract}

Keywords-RFID ; NFC; QR Codes; Image Processing

\section{INTRODUCTION}

\section{A. RFID ( RADIO FREQUENCY IDENTIFICATION)}

RFID stands for Radio-Frequency Identification. These RFID are used in form tags that use the electromagnetic fields to automatically identify and track the tag which is attached to the object. Information is stored in electronic form inside these tags.

There are two types of tags namely: Passive tags and Active tags. Passive tags collect energy from nearby RFID readers, whereas Active tags have local power source (such as battery). Unlike Barcode that need to be present in the sight of the reader, RDIF tags can be identified just by being present in the general vicinity. Due to these advantages' RFID is used for automatic identification and data capturing. These RFID can be used for tracking purposes, this can be done by fixing the RFID tag on the object that needs to be tracked.

\section{B. QR Code}

Today, QR Codes can be seen on flyers, banners, magazines, etc. You can without much of a stretch recognize these two- dimensional standardized tags around you. QR Codes let you associate with the world utilizing your cell phone. In particular, a QR Code broadens the information at removal on any physical object and make an advanced degree to advertising activities. This innovation empowers and accelerates the utilization of versatile web administrations: it is an extremely inventive advanced device.

Also, not at all like standardized identifications, which must be perused by an extraordinary scanner, numerous cell phones can check QR codes, making them a fantastic method to give a lot of data in a little space.

\section{NFC (NEAR FIELD COMMUNICATION)}

Another device used in this proposed system is NFC used to monitor the entry and exit of anyone visiting a particular place. NFC stands for Near-Field Communication. Using NFC, this system keeps a record of visitors visiting any place. This can be used for monitoring as well as maintaining a record of what the public is showing interest in. Similar to other "proximity card" technologies, NFC utilizes electromagnetic induction between two loop antennas which are attached to NFC enabled devices.

NFC tags are passive devices that can be used to read information that is stored in the tags, and in some cases can be used to also write information in the tag. Typically, in normal use the data can only be read but in some cases the data can also be rewritten. Applications of NFC includes securing personal data stored (e.g. program data ,debit and credit card, personal identification numbers (PINs), contacts). NFC tags can also be custom-encoded by their manufacturers or by using the industry specifications.

\section{LITERATURE SURVEY}

The current versions of the audio tour guide use GPS. GPS stands for Global positioning System. While it is without doubt that GPS can be very useful, it is sadly restrictive depending on its location. It is difficult to use a GPS dependent system in an indoor setting such as a museum or in a geographical location where the internet is unavailable.

Hence, we have proposed a system that uses RFID. By using a system that is dependent on Radio frequency instead of internet. We are hence eliminating the issue caused by an unstable network while using a GPS system. Using RFID also makes the system more accurate whereas 
GPS can show wrong information in a closed setting such as a museum or a remote geographical location such where the internet might not get connected. Thus by developing a system that depends on radio frequency we are building a more efficient and accurate system.

It is also our goal to provide information by initiating group interaction. Hence, we are developing a moving robot as a guide. To make the system more convenient we have developed a human following system. Since most tourist locations are extremely crowded, instead of following a human, the robot follows a QR Code. The $\mathrm{QR}$ code is a black and white pattern enclosed in a square box. This QR Code contains information in it. Each QR code is unique to each other, thus by having the robot follow the QR Code we are eliminating the possibility of the robot getting lost, or following a different group.

Finally the system is also designed to maintain log. Since most tourist locations are crowded it becomes difficult to monitor who comes to the location. To do this we have used NFC tags. NFC stands for Near Field Communication. NFC has found its use in applications such as business transactions and payments. Each individual has an NFC tag, when they tap the tag on the reader the information regarding the user gets registered in an XML sheet. Many recently released smartphones have NFC tags installed in them, thus making the use of this system more convenient.

\section{PROPOSED METHODOLOGY}

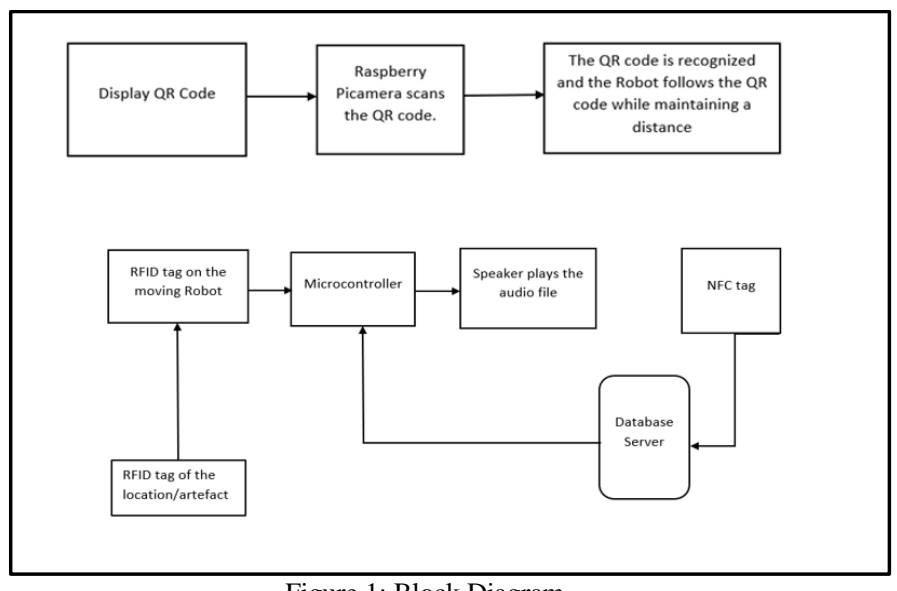

Figure 1: Block Diagram

The block diagram of the system is as shown above. As it is clearly mentioned in the above diagram, the system consists of three major parts:

- The QR Code recognition

- The RFID Communication

- NFC log maintenance

\section{A. THE QR CODE RECOGNITION}

Here we have a Raspberry Pi microcontroller with OpenCV installed in it. OpenCV is a software that uses Python language for Image processing. The microcontroller is connected to a camera. We have decided to use a Picamera, which can be replaced by a digital or High Definition (HD) camera for better reading.

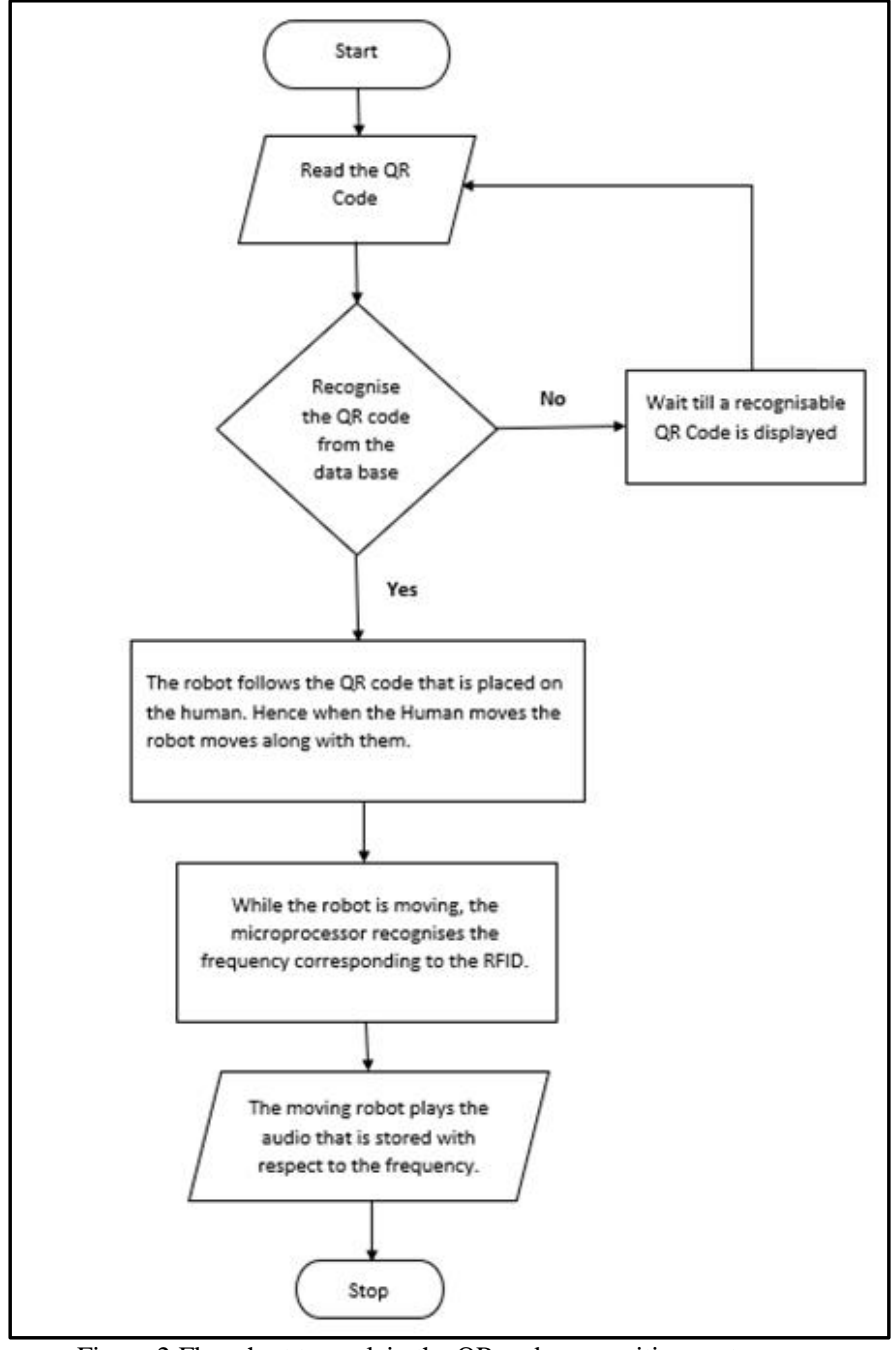

Figure 2:Flowchart to explain the QR code recognition system

As depicted in the flowchart, the camera reads the QR code and decodes it. If the recognized data is found in the memory, the robot starts following it. If the decoded data is not part of the memory, the robot waits till it reads a valid QR code. Since the application of the robot is at a tourist location, we have integrated the reading of a $\mathrm{QR}$ code to ensure that the robot follows only a particular person/group of people and does not get lost in the crowd. QR code has found its use in modern society in applications such as online transaction, payment, e-commerce, etc. Henceforth we believed it would be convenient to use the $\mathrm{QR}$ code in the commercial setting.

The QR code is displayed in front of the camera. The microprocessor executes the program and thus the QR code is decoded. The microprocessor decides an action based on two consequences. If the text decoded from the QR code is found in memory, then the servo motors start in the wheel, hence making the robot move. Here we have used a chassis with two wheels at the backend and a uni-wheel to simplify the electric connectivity of all the components. The movement of the robot is controlled by only the backend wheels. The robot follows the QR code at a constant precoded distance. The camera on the robot is rotating at a constant speed. Thus, when the person makes a turn the robot follows the person instead of stopping at the place. 
When the person moves from one location to another the robot follows the human while playing the audio file through the speaker corresponding to the artifact in the location. We have used a smartphone with an inbuilt TTS engine to globalize the system.

\section{B. RFID Communication}

The second part of the robot consists of the RFID communication. A tourist location often consists of many artifacts. In such a setting each artifact will have a unique RFID tag attached to it. Each tag will emit a unique frequency. The microcontroller will recognize the frequency being radiated, and will play an audio file through the speaker. The file to be played is stored in the memory corresponding to the frequency, thus playing the relevant audio file for the particular artifact.

We have implemented the system using NRF24L01 modules. An NRF24L01 is a single chip radio trans-receiver for a worldwide 2.4-2.5 GHZ ISM band. It consists of inbuilt frequency-synthesizer, power amplifier, crystal oscillator, demodulator, modulator, etc. It utilizes the 2.4 $\mathrm{GHz}$ band and it can work with baud rates from $250 \mathrm{kbps}$ up to $2 \mathrm{Mbps}$. Whenever utilized in open space and with lower baud rate its range can reach up to 100 meters. The power utilization of this module is simply around $12 \mathrm{~mA}$ during transmission, which is even lower than a solitary LED. The working voltage of the module is from 1.9 to $3.6 \mathrm{~V}$, however interestingly, different pins endure $5 \mathrm{~V}$ rationale, so we can undoubtedly interface it to an Arduino without utilizing any logic level converters.

\section{NFC Log Maintenance}

A maintenance system is built using NFC technology. When a NFC tag is tapped on the NFC reader(smartphone), the information regarding the user is recorded in the database. This is built to maintain a record of tourists visiting the location.

The NFC Encoder programming can likewise utilize a XML record for the NFC label encoding information instead of the Excel based document. A XML record is valuable when another framework is producing the NFC label encoding information. The simplest method to produce the underlying XML document is to make an example in the Excel record and afterward send out the XML to a nearby record. The XML would then be able to be altered by means of a content manager or potentially utilized as a layout for another framework to produce. The XML Schema Definitions (XSD) for the NFC Tags Encoding document can be downloaded from inside the NFC Encoder programming.

Hence, when a person enters a particular room or environment and taps his reader(smartphone) on the tag, thus registering his entry/exit in the $\log$ of the concerned institution.

\section{RESULTS AND DISCUSSION}

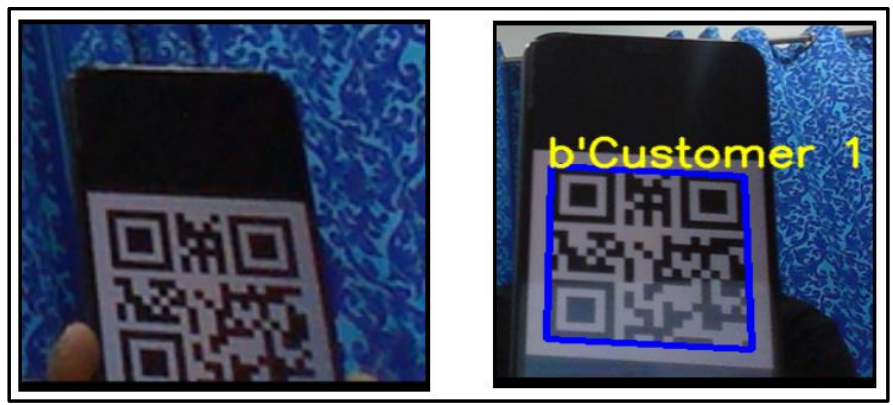

Figure 3:The figure shown above is an example of how the QR Code is recognised by the moving robot using image processing

Table 1: The table shown below lists some of the test trials and the corresponding results that were received.

\begin{tabular}{|c|c|c|}
\hline SI no & $\begin{array}{c}\text { Distance of the code from the Camera } \\
(\mathbf{c m})\end{array}$ & Response \\
\hline 1 & 5 & Identifies \\
\hline 2 & 10 & Identifies \\
\hline 3 & 15 & Doesn't identify \\
\hline
\end{tabular}

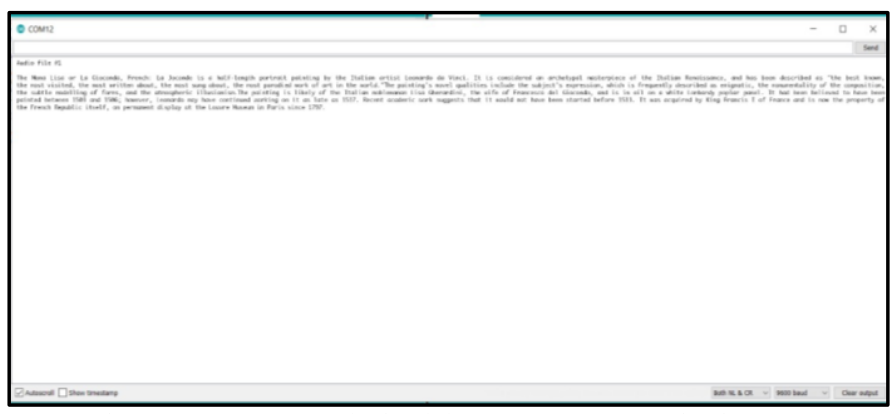

Figure 4: Text output obtained due to RFID communication

The above figure is a demonstration of how RFID communication is established between various nRF modules acting as transceivers. Here, an RF frequency corresponding to the data about a specific artefact is detected and the information about the same is displayed which is played in the form of an audio file by implementing a TTS engine. 
$<$ Row $>$

$<$ Cell $>$

$<$ Data ss:Type="String" $>$ ID Number $</$ Data $>$

$</$ Cell $>$

$<$ Cell $>$

$<$ Data ss:Type="String" $>$ First Name $</$ Data $>$ $</$ Cell $>$

$<$ Cell >

$<$ Data ss:Type $=$ "String" $>$ Last Name $</$ Data $>$

$</$ Cell $>$

$<$ Data ss:Type="String" $>$ Location $</$ Data $>$

$</$ Cell $>$

$</$ Row $>$

$<$ Row $>$

$<$ Cell $>$

$<$ Data ss:Type $=$ "Number" $>$ 13456 $</$ Data $>$

$</$ Cell $>$

$<$ Cell $>$

$<$ Data ss:Type $=$ "String" $>$ Luke $</$ Data $>$

$<$ Cell $>$

$<$ Cell $>$

$<$ Data ss:Type="String" $>$ Skywalker $</$ Data $>$

$</$ Cell $>$

$<$ Cell $>$

$<$ Data ss:Type="String" $>$ Mona Lisa $</$ Data $>$ $</$ Cell $>$

$</$ Row $>$
$<$ Row $>$

$<$ Cell $>$

$<$ Data ss:Type="Number" $>\mathbf{4 5 7 5 2 3}</$ Data $>$

$</$ Cell $>$

$<$ Cell $>$

$<$ Data ss:Type="String" > George </Data >

$</$ Cell $>$

$<$ Cell $>$

$<$ Data ss:Type="String" $>$ Washington $</$ Data $>$ $<$ Cell $>$

$<$ Cell $>$

$<$ Data ss:Type $=$ "String" $>$ Archaeology $</$ Data $>$ $</$ Cell $>$ $</$ Row $>$

Figure 5: XTML output of NFC

the above figure shows the XML output to the sample NFC communication. The ID, First Name, Last Name, Location of the user is displayed in the file.

\section{CONCLUSION}

Thus, the objective of this paper is to build a system that successfully overcomes the shortcomings of using GPS for indoor navigation and that can be used as an audio tour guide that caters to the need of a specific person ensuring personalization as well as efficiency. The use of QR code and RFID tags in a new and different way is also implemented in such a way that the construction of the system is not very complex thus leading to minimized maintenance issues. The use of RFID has erased the instability caused by the use of internet based systems. However, the system is not very efficient in recognizing and following the designated person unless they are at a detectable distance from it. Various methods involving LiFi and IR sensors could be implemented in the future to improve the performance of the system.

\section{REFERENCES}

[1] Muhammet Fatih Aslan, Akif Durdu, Kadir Sabanci ,"Shopping Robot That Make Real Time Color Tracking Using Image Processing Techniques", 2017 International Journal of Applied Mathematics, Electronics and Computers

[2] K.S. Sampada, Nithin Mathew, Pavana .A , S. Megha, Shubh Mehta, "Location Aware Audio Tour using nRF",2019 International Journal of Computer Science and Engineering, Volume 6, Issue 7, July 2018

[3] K. Sornalatha, V. R. Kavitha "IoT Based Smart Museum using Bluetooth Low Energy", 2017, 3rd International Conference on Advances in Electrical, Electronics, Information, Communication and Bio-Informatics (AEEICB17)

[4] Prof. Krishna Tripathi, Bijoy Kunnappillil, Rohit Meshram, Vicky Bhoir, "NFC- Based Attendance System ", 2016 , International Journal of Advanced Research in Computer and Communication Engineering, Vol. 5, Issue 3, March 2016

[5] SMART CELLCO, INC ., Wilmington, NC ( US), "RFID / NFC FUNCTIONALITY FOR PORTABLE ELECTRONIC DEVICES" in 2019 Patent No : US 10, 387, 870 B2

[6] Gianfranco Andìa Vera, Abanob Abdelnour, Mira Sarkis ,Apostolos Georgiadis , Darine Kaddour, Smail Tedjini, “ Passive RFID-enabled Proximity Sensor”, 2016 IEEE MTT-S International Microwave Symposium (IMS)

[7] Juraj Mihal'ov, Michal Hulič, "NFC/RFID technology using Raspberry Pi as platform used in Smart Home project", 2017 IEEE 14th International Scientific Conference on Informatics.

[8] KiBeom Kang, JeongWoo Jwa, SangDon Earl Park, "Smart Audio Tour Guide System using TTS", 2017, International Journal of Applied Engineering Research ISSN 0973-4562 Volume 12, Number 20 (2017).

[9] R.M. Nipuna Deelaka Ranasinghe, GUAN Zhen Yu, "RFID/NFC Device with Embedded Fingerprint Authentication System", IEEE, 2017 8th IEEE International Conference on Software Engineering and Service Science (ICSESS

[10] Md Sayedul Aman, Cuyler D. Quint, Ahmed Abdelgawad, Kumar Yelamarthi College of Science and Engineering, "Sensing and Classifying Indoor Environments: An IoT Based Portable Tour Guide System", IEEE, 2017 IEEE Sensors Applications Symposium (SAS).

[11] B. Lalithadevi, Annam Mallikharjuna Roa, Nagineni Dharani, A Satya Raghava, J. Buvanambigai, "Efficient Scheme for Car Parking Using RFID \&NFC", 2018, Journal of Network Communications and Emerging Technologies (JNCET), Volume 8 , Issue 4, April (2018).

[12] Romeo L. Jorda Jr.a,b, Joshua Renz A. Coballesa, Lejan Alfred C Enriqueza, Mark Lester S. Millana, Angelo J. Moraa, Melbert Neil G. Teodoroa, "Comparative Evaluation of NFC Tags for the NFCControlled Door Lock with Automated Circuit Breaker", 2018 , 2018 IEEE 10th International Conference on Humanoid, Nanotechnology, Information Technology, Communication and Control, Environment and Management (HNICEM 2018).

[13] Lei Cui, Zonghua Zhang, Nan Gao, Zhaozong Meng ,Zhen Li, "Radio Frequency Identification and Sensing Techniques and Their Applications-A Review of the Stateof-the-Art", 2019 , Sensors 2019, 19(18), 4012; https://doi.org/10.3390/s19184012 .

[14] Sankul Rathod, Priyanka Shirsat, Vaibhav Thorat, Tejaswini Panure, "Smart Attendance System using NFC and Face Recognition”, 2016, International Research Journal of Engineering and Technology (IRJET), Volume: 03 Issue: 11 | Nov -2016. 